\title{
Integración de las tecnologías de información y comunicación en la región indígena tsotsil de los altos de Chiapas *
}

\author{
Víctor del Carmen Avendaño Porras ${ }^{1}$ (1) @ \\ Estuardo Lara Ponce ${ }^{2}$ (1) @ \\ ${ }^{1}$ Centro Regional de Formación Docente e Investigación Educativa (CRESUR), México; ${ }^{2}$ Universidad \\ Autónoma Intercultural de Sinaloa (UAIS), México.
}

\begin{abstract}
Resumen. El presente artículo se centra en el estudio de las actitudes ante el uso de las TIC como una disposición aprendida que involucra procesos cognitivos y elementos conductuales. Al respecto, se trata de analizar la reacción actitudinal que los docentes de la región indígena tsotsil de los Altos de Chiapas tienen ante la integración de las tecnologías de la información y la comunicación en los salones de clase del nivel básico. Se optó por un muestreo consecutivo que intentó incluir a todos los sujetos accesibles como parte de la muestra, en donde se tuvo acceso a un grupo de 137 profesores indígenas de la zona indígena tsotsil de los altos de Chiapas, sobre un total estimado de docentes en ese territorio de 1300 . Entre los principales resultados obtenidos y descritos en esta investigación, se da muestra de que las actitudes que presentan los profesores de la región tsotsil, en general, son positivas hacia el uso de tecnologías de información y comunicación. Ello puede ser un factor que debe ser considerado en las políticas educativas con TIC que implemente el gobierno mexicano en la región.
\end{abstract}

Palabras clave: Tecnologías de la información y la comunicación, actitudes docentes, procesos cognitivos, integración de las TIC.

Integração das tecnologias de informação e comunicação na região indígena tsotsil dos altos de Chiapas

Resumo. O presente artigo centra-se no estudo de atitudes em relação ao uso das TIC como uma disposição aprendida que envolve processos cognitivos e elementos comportamentais. A este respeito, analisa-se a reação atitudinal que os professores da região indígena tsotsil dos Altos de Chiapas têm diante da integração das tecnologias de informação e comunicação nas salas de aula do nível básico. A técnica de amostragem consecutiva foi utilizadada para tentar incluir todos os sujeitos acessíveis como parte da amostra, na qual foi possível selecionar um grupo de 137 professores indígenas da área indígena tsotsil dos altos de Chiapas num total estimado de 1.300 professores neste território. Entre os principais resultados obtidos e descritos nesta pesquisa, observa-se que as atitudes apresentadas pelos professores da região de tsotsil são, em geral, positivas em relação ao uso de tecnologias de informação e comunicação. Esse pode ser um fator que deve ser considerado nas políticas educacionais com TIC implementadas pelo governo mexicano na região.

Palavras-chave: tecnologias de informação e comunicação; atitudes docentes; processos cognitivos; integração das TIC.

Integration of information and communication technologies in the tsotsil indigenous region of the highlands of Chiapas

Abstract. The present article focuses on the study of attitudes towards the use of ICT as a learned disposition that involves cognitive processes and behavioral elements. In this regard, the aim is to analyze the attitudinal reaction that the teachers of the Tsotsil indigenous region of the Highlands of Chiapas have before the integration of information and communication technologies in the classrooms of the basic level. We opted for a consecutive sampling that tried to include all the subjects that were accessible as

* La presente investigación, derivó de una estancia Posdoctoral en Educación para la Diversidad Cultural en la Universidad Autónoma Intercultural de Sinaloa, entre 2018 y 2019 , bajo la asesoría del Dr. Estuardo Lara Ponce. 
part of the sample, where we had access to a group of 137 indigenous teachers from the Tsotsil zone of the highlands of Chiapas, out of an estimated total of teachers in that area, territory of 1300. Among the main results obtained and described in this research, it is shown that the attitudes of teachers in the Tsotsil region, in general, are positive towards the use of information and communication technologies. This may be a factor that should be considered in the educational policies with ICT implemented by the Mexican government in the region.

Keywords: Information and communication technologies, teaching attitudes, cognitive processes, integration of ICT.

\section{Introducción}

Actualmente, el 59.5\% de la población en México usa Internet, siendo los estados de Baja California Sur, Sonora y Baja California las entidades que exhiben la mayor oportunidad de acceso de Internet en los hogares: $75.5 ; 71.7$ y 68.0 por ciento respectivamente, y por otra parte, los estados de Tlaxcala, Oaxaca y Chiapas exhiben la menor oportunidad de acceso a internet y otras tecnologías, con proporciones de $28.4 ; 20.6$ y 13.3 por ciento respectivamente (INEGI, 2017).

Por otra parte, las poblaciones en México prácticamente se encuentran navegando durante casi todo el día. Los momentos de hora pico de conectividad van desde las 6 a 9 horas. En las noches, los mexicanos suelen navegar entre las 21 y 24 horas. El tiempo promedio de uso total del Internet es de 8 horas con 12 minutos (Tamayo, 2018).

La presencia de los contextos indígenas en internet es aún menor. Al respecto, cabe destacar, en los estudios de Quero y Madueño (2006), Quinchoa (2011), Villacrés (2016), la sub representación de estos colectivos en el cotidiano de las redes que se ha reflejado para la nación. Una cuestión importante que cabe resaltar es que, en Chiapas en general y en la zona del estudio que se presenta a continuación (los altos de Chiapas), las herramientas tecnológicas están al alcance de la mayor parte de la población, hablando de teléfonos celulares, equipos de cómputo e internet, en esta región incluyen varias zonas o comunidades en las que la conectividad no está habilitada, lo cual obstaculiza el empleo de los diferentes recursos.

El gobierno de México, a través de la Secretaría de Educación Pública, está concernido en cuanto a la mejora de la competencia digital de los estudiantes (referencia) y está consciente, siguiendo las recomendaciones de la UNESCO (2004), de que los profesores son una pieza clave para la integración de las TIC en las escuelas. Pareciera que este proceso de integración 
ha sido lento y disímil; produciendo una brecha significativa, pues las TIC han sido adoptadas con mayor soltura en el ámbito escolar administrativo que en la práctica docente (referencia).

En cualquier caso, a pesar del interés de los profesores, existen problemas técnicos en la región.

Por otra parte, también ocurre que, según estudios de diversos autores, como Area (2002), Cabero (2004), Cebrián (1997), e Imbernón (1994), son los docentes de nivel superior quienes se encuentra mayor equipados de tecnologías para impartir sus clases.

Los estudios citados sobre la apropiación de la competencia digital en contextos indígenas ponen de manifiesto el interés de las comunidades por preservar su cultura en el tiempo de internet, en particular sus tradiciones y escritura, si bien se está produciendo un proceso de aculturación.

Al respecto, Quero y Madueño (2006), en su estudio evaluativo sobre un programa para el uso de TIC en escuelas indígenas, en concreto, que desarrollaron y aplicaron un software educativo contextualizado en una comunidad de la cultura wayuu, para estimular la lectura y escritura del Wayuunaiki. Estas tecnologías consiguen ser manejadas en apoyo a los planes educativos que plantea el Régimen de Educación Intercultural Bilingüe Mexicano. Los resultados demostraron que los niños y los docentes manifestaron interés al utilizar software en su idioma.

Por su parte, Quinchoa (2011) realiza un estudio antropológico que aborda el uso de la tecnología en las escuelas rurales. Por medio de herramientas etnográficas, detalla la producción de enlaces y la generación de un portal web del resguardo indígena de Puracé, departamento del Cauca, y analiza, a su vez, cómo conciben los Kokonucos la tecnología.

Por último, Villacrés (2016) aborda un estudio sobre la aculturación, analizando el uso de las TIC como cultura digital que transgrede en la cultura Kichwa. El autor, que desarrolla un estudio de investigación mixta, analiza las tradiciones, costumbres y lenguas de los pueblos indígenas ancestrales de Latinoamérica, concluyendo que las poblaciones estudiadas hacen uso del internet y de las tecnologías de comunicación; y las comunidades rurales cuentan con dotación tecnológica suficiente para mantener contacto a través de internet y teléfonos inteligentes, además de insinuar que la asimilación de la cultura digital en este grupo étnico es un resultado trasversal derivado de proceso de aculturación de la cultura mestiza sobre la indígena. 
Dar respuesta a la exigencia académica de generalizar el uso de las TIC en el aula, junto a promover la atención de las disfunciones que la inmersión en internet trae a los contextos indígenas, se convierte en una de las motivaciones centrales para estudiar la actitud del docente de educación básica de una zona indígena -en cualquiera de sus roles profesionales de asesor, tutor y mediador-, pues son componentes que afectan el proceso y la calidad de la enseñanza (referencia) ; ya que el docente, en su papel de asesor y tutor, motiva y provoca, a través de su ejemplo, el uso de dichas herramientas como andamio en el proceso de construcción del conocimiento y aprendizaje.

Las actitudes intervienen de forma concluyente sobre nuestra inclinación individual, manifestándose en emociones, evocaciones, credos y acciones, entre otros elementos, como parte de la interacción con otros individuos (referencia).

Además, las actitudes, al igual que el propio proceso de aprendizaje, no son estáticas, sino que se transforman y se pueden ver perturbadas y reformadas a lo largo del tiempo, influenciadas por personas, prácticas y contenidos (Herdina y Jessner, 2002).

\section{Diseño}

La mayoría de las investigaciones sobre análisis de actitudes usa cuestionarios, entrevistas y escalas. De estos métodos usados el que posee mayor importancia científica por ser el más probado, establecido, diferenciado y usado, es el de las escalas (Vallejo, Urosa y Blanco, 2003).

Las escalas son distintas tanto en su configuración como en su construcción, pero concuerdan en un objetivo: conceder a un sujeto una posición numeral, misma que muestra el valor de la actitud hacia un objeto categórico, en este caso, hacia las TIC (Tejedor, García-Valcárcel Muñoz-Repiso y Prada San Segundo, 2009).

Dado que en el contexto de esta investigación se pretende analizar las actitudes de los docentes ante el uso de las TIC en docentes de la zona indígena tsotsil de los altos de Chiapas, se adaptó la escala construida por Tejedor y otros (2009), misma que contiene ítems validados y confiables en torno al concepto de "actitud", como son:
1. La perspectiva cognitiva
2. La perspectiva afectiva
3. La perspectiva conductual 
Con el objeto de llevar a cabo la recogida de información, se preparó el instrumento con los ítems que proponen estos autores. Se eligió a los sujetos mediante un muestreo consecutivo que intentó incluir a todos los sujetos accesibles como parte de la muestra, en donde se tuvo acceso a un grupo (137 profesores indígenas tsotsiles de la región altos de Chiapas, sobre un total estimado de docentes en los altos de Chiapas de 1300) en un determinado intervalo de tiempo (mayo y junio de 2018), para llevar a cabo el estudio. Todos ellos son docentes de educación básica de la zona indígena tsotsil de los altos de Chiapas.

El instrumento para obtener la información fue un cuestionario de actitudes, válido y fiable, que consta de 30 ítems valorados en una escala Likert, con un nivel de respuesta entre 1 y 5 :

- Muy de acuerdo

- De acuerdo

- Indiferente

- Desacuerdo

- Muy en desacuerdo

Los ítems que integraron el cuestionario tuvieron el propósito de medir las actitudes docentes ante la aplicación de las TIC de los docentes de educación básica de la zona indígena tsotsil de los altos de Chiapas.

Los ítems son claros, valorativos y envuelven un único tópico, el del uso de las TIC en el aula, sin embargo, se agruparon en cuatro categorías que intentaron hacer un cruce por aquellos aspectos que atienden el marco actitudinal de los docentes en relación con las TIC.

Estas dimensiones reconocen a los siguientes aspectos:

- La actitud ante la innovación metodológica impuesto por el uso de las tecnologías.

- La actitud ante la combinación de las tecnologías a las asignaturas que imparten.

- La actitud ante la consecuencia de las tecnologías en el proceso de aprendizaje

- La actitud ante los contextos de acceso, conectividad a internet, y otros. 
Previo a la aplicación del cuestionario, y no obstante la validación del mismo que está claramente descrito por Tejedor y otros (2009), se procedió a la validación del instrumento en el contexto en que se llevó a cabo el estudio. Esto se hizo así por tratarse de un instrumento aplicado en un contexto totalmente distinto al que fue diseñado originalmente. Se procedió de la siguiente manera:

Se definió la variable

a) El instrumento fue valorado por un grupo de jueces, profesores de la Escuela de Psicología, de la Universidad Mesoamericana, de San Cristóbal de las Casas. Para llevar a cabo dicha valoración, se presentó el instrumento a 9 jueces que valoraron el grado de pertinencia de cada ítem.

b) Se fijó el número de ítems, por la puntuación obtenida de cada uno de ellos, por lo que se tomaron los 30 que obtuvieron la más alta calificación.

c) El cuestionario lo respondieron 59 mujeres y 78 hombres de edades entre los 25 y 44 años mayormente, todos con estudios de licenciatura y 62 con estudios de posgrado.

\section{Descripción y análisis de resultados}

Los datos obtenidos mediante la integración del instrumento aportan un resultado empírico favorable, y demuestran que resulta necesario y de gran importancia analizar las actitudes docentes frente a la utilización de las tecnologías de la información y comunicación en la región indígena tsotsil de Ios altos de Chiapas. A partir del cuestionario aplicado, se obtuvieron diversos resultados que se describen a continuación.

Como se muestra en la figura 1,8 de cada 10 maestros (el $86 \%$ de los encuestados) están totalmente de acuerdo o de acuerdo con la aseveración de que "las TIC fomentan la implicación en los métodos de enseñanza y aprendizaje". El desacuerdo y la indiferencia son muy pequeños. 


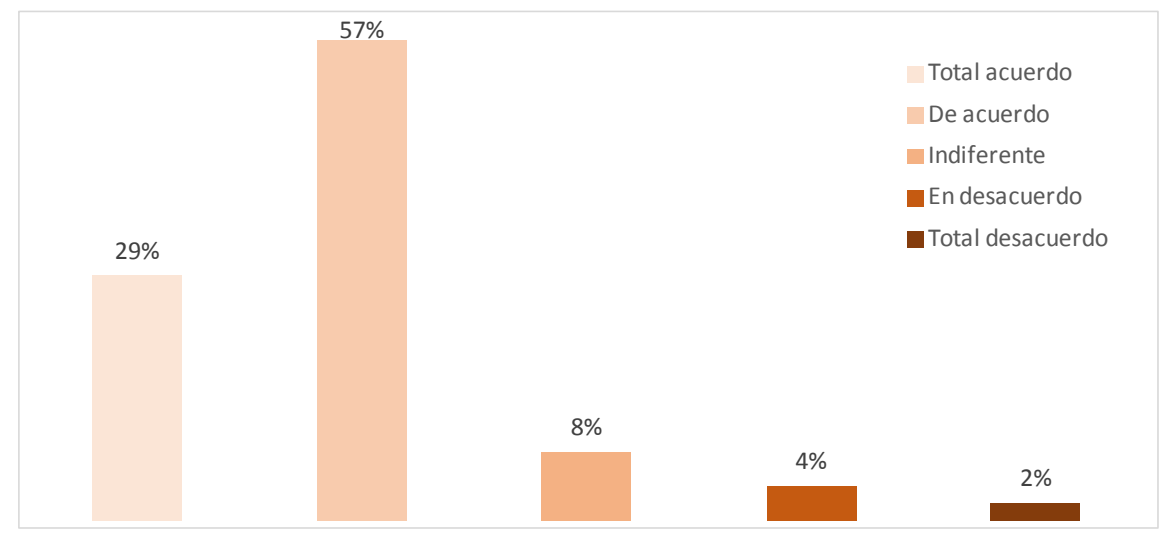

Figura 1. Las TIC fomentan la implicación en los procesos de enseñanza y aprendizaje.

Fuente: Elaboración propia

Respecto al enunciado "Ios profesores deben utilizar las TIC para mejorar la calidad de los procesos de aprendizaje", se obtuvo que un $91 \%$ está de totalmente de acuerdo o de acuerdo, tal como se muestra en la figura 2.

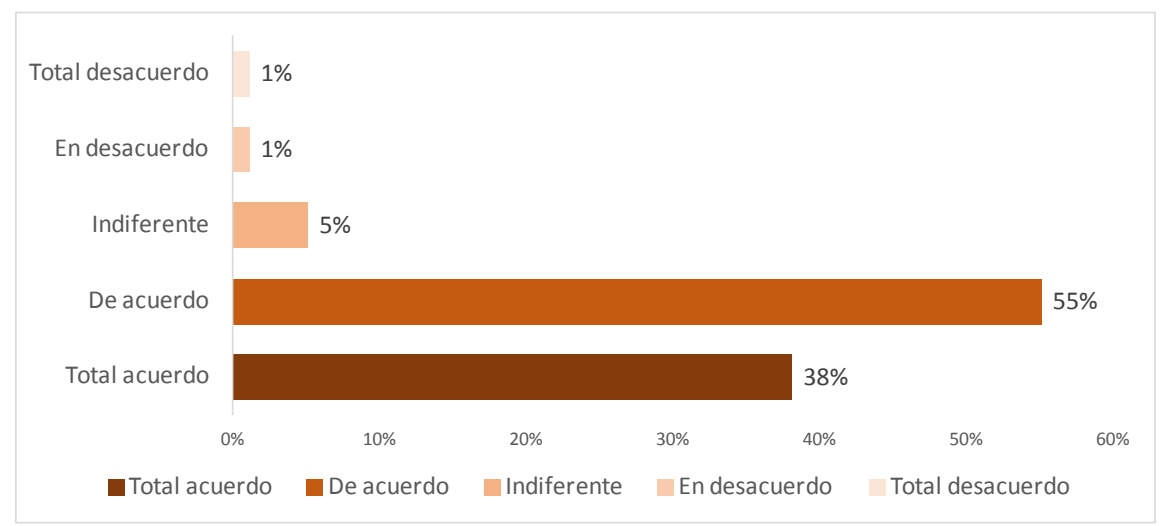

Figura 2. Los profesores deben utilizar las TIC para mejorar la calidad de los procesos de aprendizaje.

Fuente: Elaboración propia

En la figura 3 se incluyen los resultados obtenidos sobre el enunciado" es imprescindible incorporar las TIC en las aulas", acerca de lo cual se obtuvo un $91 \%$ de respuestas en la opción total acuerdo y de acuerdo. 


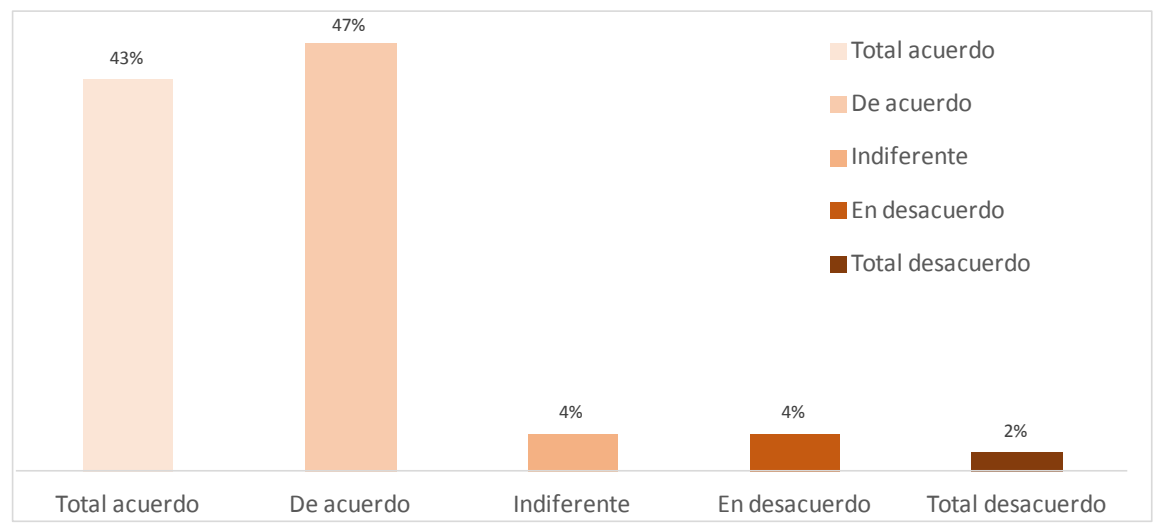

Figura 3. Es imprescindible incorporar las TIC en las aulas.

Fuente: Elaboración propia

En la figura 4, se observa que el $86 \%$ está de totalmente de acuerdo o de acuerdo con que "las clases mejoran a medida que se van incorporando las TIC".

Figura 4. Las clases mejoran a medida que se van incorporando las TIC Fuente: Elaboración propia.

En la siguiente gráfica se describen los resultados de la afirmación "las TIC permiten la consecución de las competencias" en la que el $81 \%$ de los encuestados respondieron estar totalmente de acuerdo o de acuerdo. 


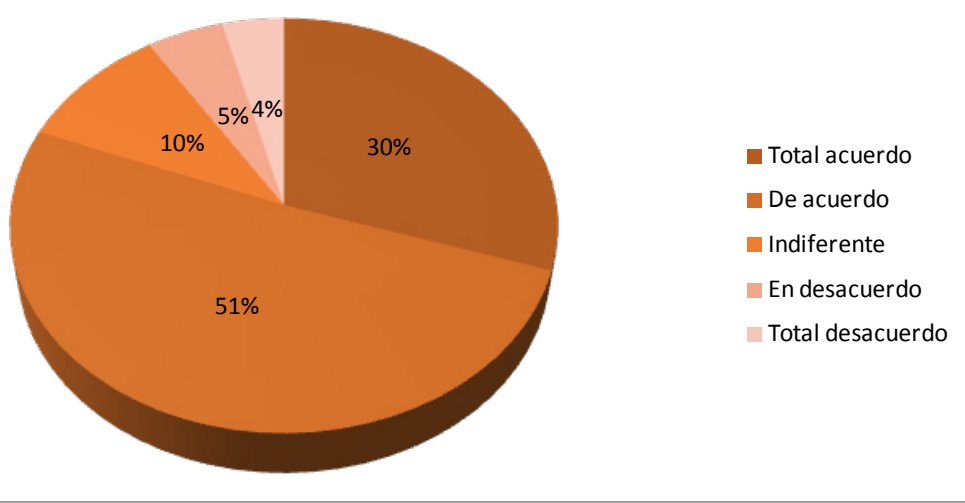

Figura 5. Las TIC permiten la consecución de las competencias.

Fuente: Elaboración propia

En la figura 6 se describen los resultados que se obtuvieron a partir del ítem que recupera la identificación por parte de los encuestados del nivel de conocimiento que poseen sobre diversas herramientas tecnológicas como el programa Word, Power Point, entre otras. En este sentido el 67\% de los participantes mencionan tener un muy alto 0 alto conocimiento.

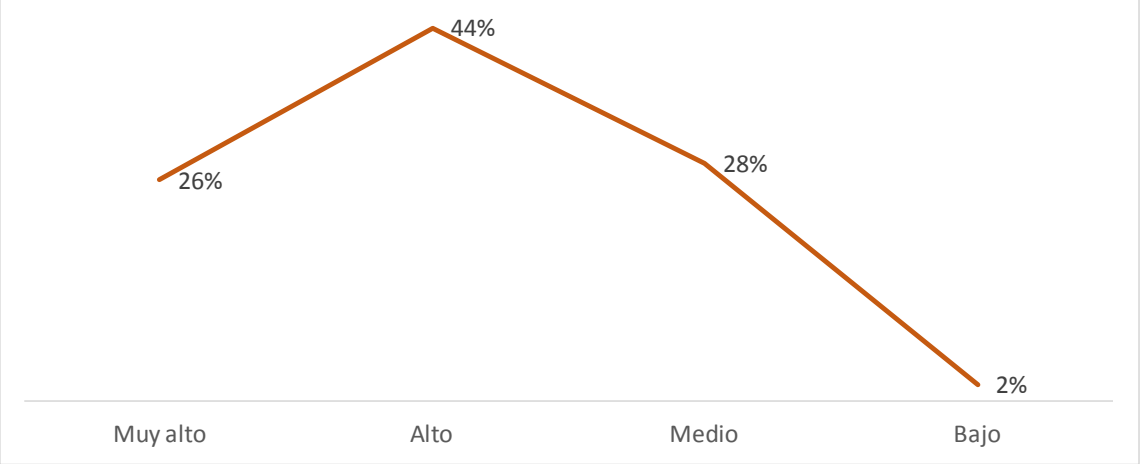

Figura 6. Nivel de conocimiento sobre herramientas de usuario y programas básicos como: Word, PPT, otros.

Fuente: Elaboración propia.

La siguiente gráfica da cuenta de cómo los encuestados conciben el nivel de conocimiento que poseen respecto a buscadores de información como Google y Yahoo, entre otros. Los resultados dan cuenta de que el $65 \%$ menciona tener un muy alto o alto conocimiento sobre ellas. 


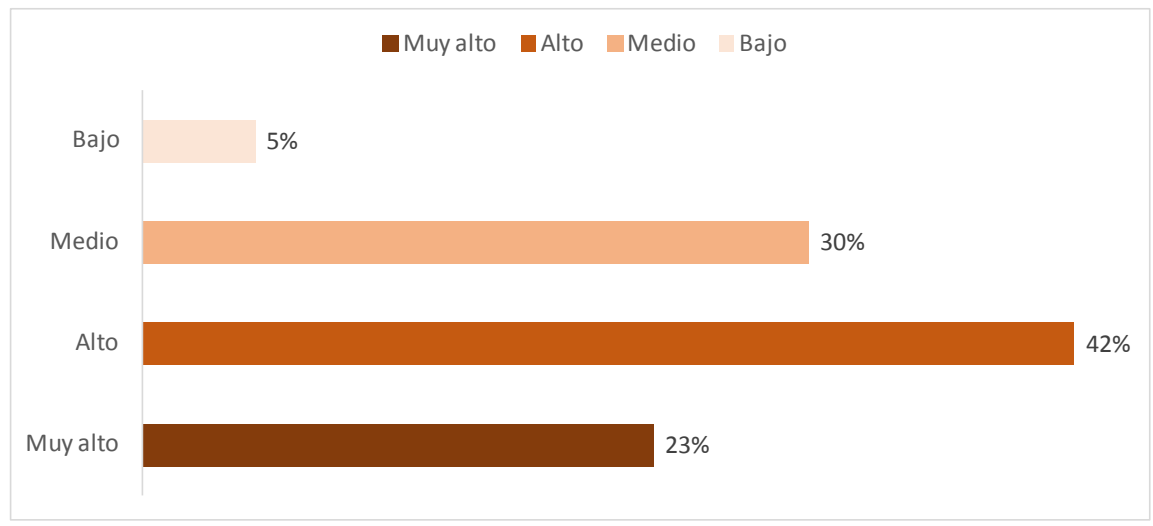

Figura 7. Nivel de conocimiento sobre buscadores de información en red.

Fuente: Elaboración propia.

En seguida se describe los resultados acerca del nivel de conocimientos que poseen los participantes sobre los sistemas de comunicación como foros, chat y videoconferencias, a este respecto (figura 8) el 57\% menciona poseer un muy alto $o$ alto conocimiento.

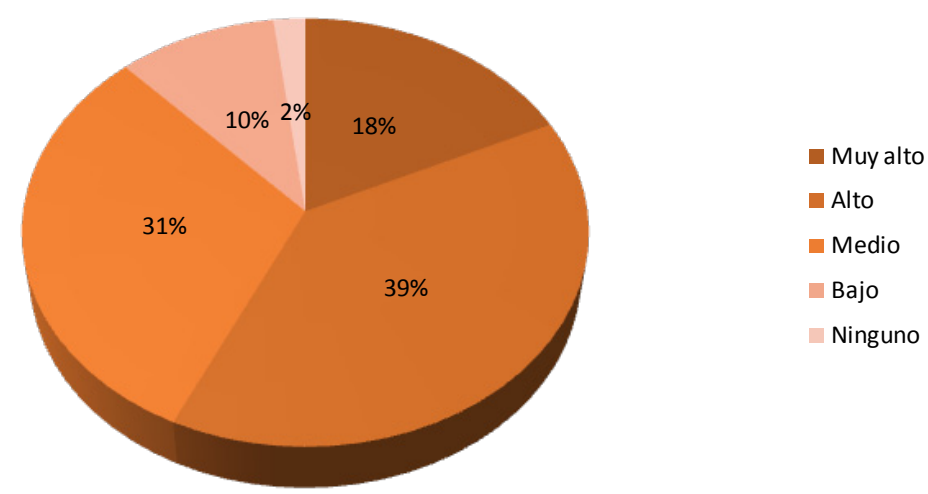

Figura 8. Nivel de conocimiento sobre sistemas de comunicación como: foros, chat y videoconferencias.

Fuente: Elaboración propia

A continuación, en la figura 9, se detallan los resultados sobre el nivel de conocimiento que los participantes mencionan tener sobre plataformas virtuales para la enseñanza-aprendizaje como Sakai, Moodle y Suma, entre otras. A este respecto, sólo el 33\% afirma que tiene un nivel muy alto 0 alto de conocimiento. 


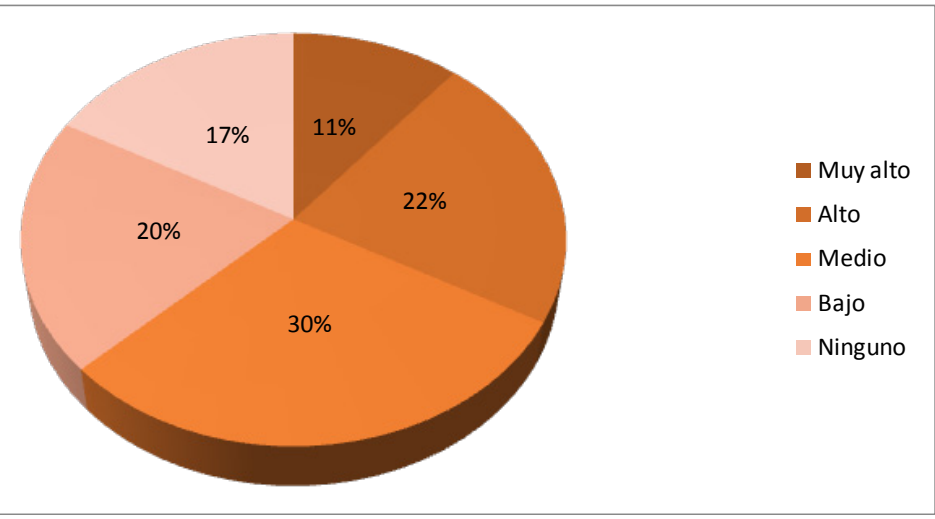

Figura 9. Nivel de conocimiento sobre plataformas virtuales de enseñanza-aprendizaje como: Sakai, Moodle y Suma, etc.

Fuente: Elaboración propia.

Por último se detallan los resultados respecto a la creación de materiales virtuales y el uso de recursos en red para la enseñanza y el aprendizaje que hacen los participantes utilizando, por ejemplo el portafolio de evidencias electrónico, la web didáctica, las wikis o los videojuegos. A este respecto la mayoría de los encuestados, el 44\%, respondieron que siempre o frecuentemente hacen uso de estas herramientas.

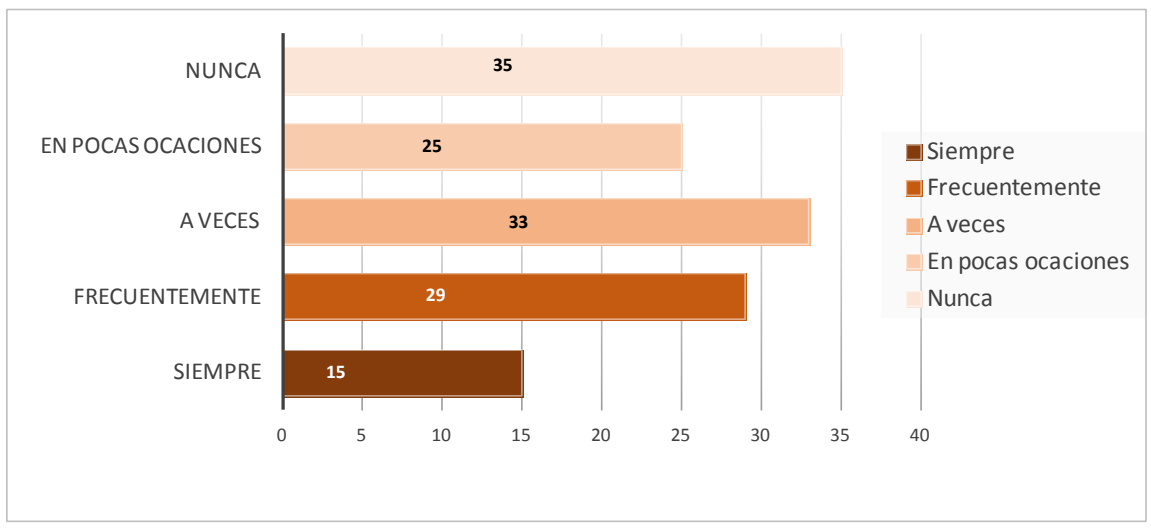

Figura 10. Uso que realiza de tecnologías como: Portafolio electrónico, Web didáctica, Wiki, videojuegos, etc.

Fuente: Elaboración propia. 


\section{Discusión}

Se ha mencionado hasta ahora la importancia de la implementación de las TIC en la profesión docente y las actitudes que presentan frente a estas tecnologías. Sin embargo, es importante señalar que, en las últimas décadas se han producido cambios importantes relacionados con la formación, actualización y desempeño de los docentes.

En este sentido, el concepto de calidad en el desempeño docente debe considerarse en el presente estudio, ya que es un concepto que puede aplicarse a cualquier elemento que configure el quehacer docente. Podemos hablar de calidad en el desempeño de la profesión docente, en los aprendizajes, en las estrategias que se implementan, en los objetivos que se plantean, en los indicadores que se diseñan o bien en los resultados académicos.

Ahora bien, existen modelos y enfoques que cuentan con una estructura definida que permiten evaluar el desempeño docente con parámetros previamente establecidos (Aldana, 2003).

Entre los enfoques más representativos figura el de Valdés y otros (2011) en el que, de acuerdo con el énfasis o centro de atención, caracteriza cuatro tendencias de los modelos de evaluación de desempeño: "modelo centrado en el perfil del maestro, modelo centrado en los resultados obtenidos, modelo de la práctica reflexiva y, por último, el modelo centrado en el comportamiento del docente en el aula".

Considerando las respuestas del instrumento aplicado con anterioridad, nos enfocaremos al último modelo centrado en el comportamiento del docente en el aula, ya que las categorías que se analizan están relacionadas con los aspectos que se considera en este.

En el modelo centrado en el comportamiento del docente en el aula de Valdés y otros (2011), se hace hincapié en la forma en cómo el docente lleva a cabo las actividades de aprendizaje, cómo trabaja, su dedicación y compromiso frente a la inclusión de las TIC, que indiscutiblemente determina los resultados, es decir, este modelo se apoya en identificar la capacidad del docente para crear un ambiente favorable para el aprendizaje en el aula.

Por otra parte, existen disyuntivas respecto a las críticas sobre el modelo, por un parte, puede subestimarse el desempeño y el producto del docente y por otra parte, se está vulnerable hacia la subjetividad de la evaluación del desempeño docente, ya sea por parte del estudiante o bien por los entes evaluadores del desempeño docente. 
Los resultados que se presentan, dan cuenta de la percepción y la actitud de los docentes frente a las TIC, desde su valorización personal, desde su perspectiva como docentes y desde su posición como profesores frente a grupo y su autoevaluación de desempaño.

De acuerdo a lo anterior, y a partir de los resultados obtenidos con el instrumento aplicado, se puede inferir que las actitudes que muestran la mayoría de los docentes son positivas pues los más altos porcentajes (del 80 al $90 \%$ o más) se concentran en la respuesta de acuerdo a las siguientes categorías:

- Las TIC fomentan la implicación en los procesos de enseñanza y aprendizaje,

- Ios profesores deben utilizar las TIC para mejorar la calidad de los procesos de aprendizaje,

- es imprescindible incorporar las TIC en las aulas,

- las clases mejoran a medida que se van incorporando las TIC,

- las TIC permiten la consecución de las competencias.

Así, los resultados demuestran, por un lado, que de manera general los docentes poseen actitudes positivas hacia las TIC, especialmente en cuanto a la integración de éstas en su práctica en el aula y en el desarrollo de estrategias; además de mostrar posibilidades de interacción con las mismas.

Por otra parte, respecto al nivel de conocimiento que manifiestan tener los profesores en el empleo y la utilización de las TIC y de los diferentes recursos se obtuvo un alto porcentaje, a saber:

- Nivel de conocimiento sobre herramientas de usuario y programas básicos como:

- Word, PPT, Excel (67\%),

- nivel de conocimiento sobre buscadores de información en red $(65 \%)$,

- nivel de conocimiento sobre sistemas de comunicación como: foros, chat y videoconferencias (57\%).

En relación a estas categorías que tienen que ver con el nivel de conocimiento sobre herramientas y uso de TIC, se denota que los docentes se sienten cómodos y familiarizados con herramientas de propósito general de la informática clásica, asociadas al funcionamiento principal de las computadoras, con herramientas de búsqueda y de comunicación. Se trata 
de 3 componentes de la competencia digital básica en los que se sienten cómodos y que pueden estar contribuyendo a su percepción positiva de la integración de las TIC.

Ahora bien, respecto al nivel de conocimiento sobre plataformas virtuales de enseñanza-aprendizaje como Sakai, Moodle y Suma, es Ilamativo el descenso de conocimiento de estas herramientas para resolver problemas (en este caso educativos). Sólo 1 de cada 3 profesores parece sentir que tiene conocimientos avanzados.

Estos porcentajes sugieren que los profesores, a pesar de que el uso de las plataformas educativas ha ido en aumento, también en la región en cuestión, no se muestran predispuestos a su implementación.

Los resultados obtenidos respecto al uso que realizan de tecnologías como: portafolio electrónico, Web didáctica, Wiki y videojuegos, entre otros, los resultados son similares.

La situación de alfabetización de esta población, ya que según el Programa Regional de Desarrollo (2013-2018), de la población de 15 años y más, $26.1 \%$ es analfabeta, $76.12 \%$ tiene algún grado de estudios, de la cual $33.68 \%$ tiene primaria completa, $23.48 \%$ primaria incompleta, $20.71 \%$ secundaria completa y $21.20 \%$ educación pos básica y $0.35 \%$ no lo especifica. Cabe sugerir que el promedio de escolaridad es de 4.6 años, con 5.3 años para los hombres y 3.9 para las mujeres.

En consecuencia de lo anterior, es importante considerar que estos porcentajes pudiesen influir en el nivel de comprensión y utilización de los recursos que se han mencionado, aunado a otro factor, el sociolingüístico, puesto que según los datos del INEGI (2018), las lenguas indígenas más habladas en el estado de Chiapas son:

\begin{tabular}{cc}
\hline Lengua indígena & Número de hablantes (año 2010) \\
\hline Tzeltal & 461236 \\
Tzotzil & 417462 \\
Chol & 191947 \\
Zoque & 53839 \\
\hline
\end{tabular}

Fuente: Estadísticas del INEGI (2018). 
En Chiapas, hay 1,141,499 personas mayores de 5 años que hablan alguna lengua indígena, lo que representa $27 \%$ de la población del estado y de cada 100 personas que expresaron hablar alguna lengua indígena, 14 no hablan español.

Es una cuestión que puede obstaculizar el uso de las tecnologías enfocadas en la educación, ya que el idioma puede ser una causal de no poder incluirlas en dicha práctica, no tanto para los profesores, sino para los estudiantes a quienes van dirigidas las actividades, esto implicaría realizar una adecuación en los contenidos que se incluyan en dichas actividades, así como un esfuerzo mayor para la instrucción con las TIC.

\section{Conclusiones}

Antes de comenzar, conviene indicar que la realización del estudio es una oportunidad de auto-evaluación, por parte de los docentes, para la mejora de la integración de las TIC en las escuelas de contexto indígena.

Los resultados obtenidos y descritos en esta investigación dan muestra de que las actitudes que presentan los profesores de la región tsotsil, en general, son positivas hacia las computadoras. Ello puede ser un factor que debe ser considerado en las políticas educativas con TIC que implemente el gobierno mexicano en la región.

Sin embargo, para que pueda existir un verdadero impacto de las TIC en la práctica y en el proceso de enseñanza - aprendizaje, se requiere de una visión integradora, partiendo de la percepción de los profesores sobre su práctica y el empleo de las TIC.

La baja confianza en sus conocimientos puede obstaculizar la realización de prácticas que incluyan la inserción y el apoyo con las TIC.

Al respecto, es llamativa la ambivalencia entre el entusiasmo que suscitan determinados dispositivos (asociados a algunos componentes de la competencia digital más tradicional) y el escaso conocimiento de aquellos que pueden transformar esos entornos.

En ese sentido, no se puede dejar de lado la importancia de la incorporación de las TIC al currículo de la carrera docente, como contenido y como un eje transversal, particularmente de herramientas TIC que permitan configurar entornos de aprendizaje avanzados. 
A partir del análisis de las actitudes que se manifiestan, se puede ir recuperando las inquietudes y necesidades que ellos mismos refieren 0 presentan, así mismo se pueden encontrar propuestas profesionales que reconozcan a los nuevos desafíos, duelos y requerimientos a los que se enfrenta actualmente la profesión docente.

Es esencial tomar estos resultados preliminares y convertirlos en objetivos hacia la mejora continua, es decir, buscar las estrategias necesarias y pertinentes para fortalecer la calidad académica de los profesores del nivel básico, implementando propuestas integrales para los procesos de enseñanza y aprendizaje que les permita desarrollar habilidades, conocimientos y capacidades para la innovación en la educación, fortaleciendo el conocimiento a través de didácticas efectivas, eficaces y eficientes, en donde se integren las necesidades requeridas por los docentes de la región.

La realidad, es que el sólo hecho de estudiar las actitudes de los profesores, abre la posibilidad de conocer las necesidades que presentan y de potencializar sus habilidades, que solo constituye el inicio de un trayecto académico interminable.

\section{Referencias bibliográficas}

Aldana, I. A. M. (2003). Evaluación del desempeño docente: fundamentos, modelose instrumentos. Coop. Editorial Magisterio.

Area, M. (2002). Igualdad de oportunidades y nuevas tecnologías. Un modelo educativo para la alfabetización tecnológica. Educar, 29, 55-65.

Cabero, J. (2004). Formación del profesorado en TIC. El gran caballo de batalla. Comunicación y Pedagogía: Nuevas tecnologías y recursos didácticos, 27-31.

Cebrián, M. (1997). Nuevas competencias para la formación inicial y permanente del profesorado. Edutec. Revista electrónica de Tecnología educativa, 6.

Herdina, J. y Jessner, U. (2002). A dynamic model of multilingualism: Perspectives of change in psycholinguistics (Vol. 121). Multilingual Matters.

Imbernón, F. (1994). La formación del profesorado. Buenos Aires: Paidós.

INEGI. (2018). Disponibilidad y uso de TIC. https://bit.ly/2FjKeg2

Quero, S. y Madueño, L. (2006). Süchiki Walekerü: un ejemplo del uso de las TIC en escuelas indígenas. Caso Wayuu. Educere, 10(34), 435-442.

Quinchoa, W. (2011). Apropiación y resistencia social de las TIC en el resguardo indígena de Puracé, Cauca, Colombia. Revista iberoamericana de ciencia tecnología y sociedad, 6(18), 241-257. 
Tamayo, J (2018). Hábitos de Usuarios de Internet en México 2018. https://bit.ly/2JYDUtD

Tejedor, F; García-Valcárcel Muñoz-Repiso, A. y Prada San Segundo, S. (2009). Medida de actitudes del profesorado universitario hacia la integración de las TIC. Comunicar: Revista científica iberoamericana de comunicación y educación, 33, 115-124.

UNESCO. (2009). Medición de las tecnologías de la Información y la comunicación (TIC) en educación - Manual del usuario. Montreal: UNESCO-IEU. https://bit.Iy/2T4rcBi

Valdés, A; Olivarría, A; Gabriela, C; Angulo, J; Martínez, C; Alonso, E. y García, R. (2011). Actitudes de docentes de educación básica hacia las TIC. Magis. Revista Internacional de Investigación en Educación, 3(6), 379- 392.

Vallejo, P; Urosa, B. y Blanco, Á. (2003). Construcción de escalas de actitudes tipo likert. Madrid: La Muralla.

Villacrés, J. (2016). Incidence of the Use of Information and Communication Technologies (ICT) on Cultural Traditions of Kichwa Youths in Ecuador. Asian Journal of Latin American Studies, 29(1), 109-131 